\title{
Contribution à l'inventaire des Hyménoptères Anthophila du département du Lot : liste préliminaire commentée
}

par Christophe PHILIPPE*

Publication : juillet 2020

Résumé. Cet article présente une première liste commentée des abeilles (Hymenoptera: Anthophila) du département du Lot (46). Elle présente 398 espèces dont 111 Apidae, 82 Halictidae, 79 Megachilidae, 92 Andrenidae, 27 Colletidae et 7 Melittidae. L'article indique, pour chaque espèce, certains traits de vie écologiques (lectisme, socialité, nidification) et des données d'occurrence au cours de 3 périodes historiques, ainsi que leur statut de rareté en lien avec la liste rouge UICN des abeilles d'Europe mais en lien avec leur rareté à l'échelle départementale. Une comparaison est établie avec la faune française des Anthophila. Enfin, le caractère patrimonial de chaque espèce est évalué et une présentation des espèces remarquables d'Anthophila pour le département du Lot est effectuée.

Mots-clés. Hyménoptères, Anthophila, inventaire, France, Lot.

Abstract. Contribution to the inventory of Hymenoptera Anthophila of the Lot department: a commented preliminary list. This article presents a first commented list of solitary bees (Hymenoptera: Anthophila) from the Lot department (46). The list presents 398 species including 111 Apidae, 82 Halictidae, 79 Megachilidae, 92 Andrenidae, 27 Colletidae, 7 Melittidae. After a short presentation of the department, the article continues, for each species, with a presentation of their occurrence and their ecological traits (lectism, sociality, nesting). Through 3 major historical periods the rarity status of each species is added and compared to the IUCN red list of European bees; a rarity index at the departmental scale is also provided. A comparison is made with the French fauna of Anthophila. Finally, a focus on some of the remarkable species of Anthophila of the Lot department is provided.

Keywords. Hymenoptera, Anthophila, inventory, France, Lot.

\section{Introduction}

La faune des hyménoptères Anthophila du Lot ${ }^{1}$ n'a fait l'objet d'aucun travail de synthèse jusqu'à présent. Cependant, deux publications concernent la région élargie et un département limitrophe ; ce sont respectivement celle de PÉREZ (1890) - un catalogue des abeilles du grand Sud-Ouest y recensant 491 espèces -, et celle de BALITEAU et al. (2013), qui correspond à une liste des espèces de l'Aveyron (199 recensées). La présente liste a été réalisée grâce à des données anciennes issues de récoltes de Gérard CANOU (avant 1965) et de Marc \& Hubert Tussac (entre 1965 et 1999) : leur collection personnelle comprenait quelques centaines de spécimens d'Anthophila très aimablement transmis à l'auteur. Enfin, des données plus récentes provenant de plusieurs membres de l'Observatoire des Abeilles et de nombreuses prospections entreprises par l'auteur du printemps 2010 à l'été 2018 par chasse à vue, fauchage et capture statique (coupelles colorées, pièges jaunes), ont été compilées. Cette liste comprend 398 espèces d'Anthophila, parmi lesquelles on trouve certaines espèces de la Liste rouge européenne UICN (NIETO et al. 2014).

Présentation du département et des localités prospectées

La situation géographique du département du Lot, sa géologie variée, son relief (altitude de 100 à

\footnotetext{
* 15 rue de l'Auxerrois Bégoux, 46000 Cahors - c.phil24@orange.fr

1 Département du Sud-Ouest (46) inclus dans la région Midi-

Pyrénées avant de l'être en Occitanie
} 
$780 \mathrm{~m})$ et ses influences climatiques diversifiées (méditerranéenne, atlantique et montagnarde) déterminent la grande diversité des territoires qui le composent.

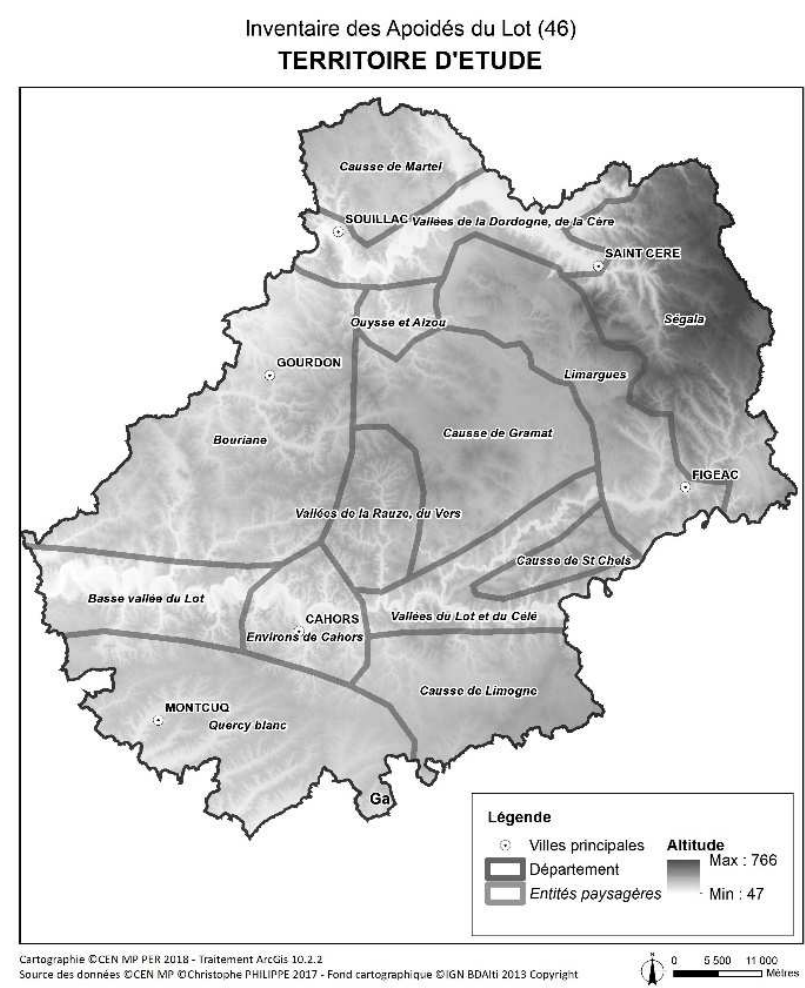

Carte I. Territoire d'étude.

Du Nord-Est au Sud-Ouest se succèdent les petites régions suivantes, caractérisées par une géologie et une végétation particulière (carte I) (ANONYME, 2020) :

1) À l'est, le Ségala est constitué de collines et de plateaux situés à 300-700 m d'altitude, fortement entaillés par les cours d'eau principaux (Lot et ses affluents). Les vallées sont encaissées (pentes fortes à très fortes), les affleurements rocheux, abrupts et les influences climatiques, montagnardes et atlantiques ; le substrat est typique des terrains cristallins (micaschistes et schistes, gneiss à grain fin, schistes gréseux, quelques grès et de rares quartzites) ; selon que l'on se trouve sur les collines et plateaux ou dans les vallées, dominent respectivement la forêt (châtaignier, pin sylvestre, sapin de Douglas et mélèze $\mathrm{y}$ occupent une plus grande superficie que les hêtres, chênes rouvres et bouleaux), ou l'élevage et la polyculture (mosaïques paysagères composées de prairies, cultures souvent fourragères, haies bocagères, tourbières et quelques bois).
2) La Limargue est une zone de 6 à $14 \mathrm{~km}$ de large qui sépare le Ségala des Causses.

3) Les Causses couvrent les deux tiers du département et apparaissent globalement comme de grands plateaux ondulés situés à 300-400 m d'altitude. Dans les zones hautes constituées d'affleurements rocheux sur lithosols calcaires, on trouve des bois (chênaie pubescente claire) et des pelouses herbacées. Viennent ensuite des zones intermédiaires à faible pente aux sols superficiels plus continus, les rendzines rouges (sols argileux peu profonds rouges sur dalle calcaire fissurée y laissant des éclats) ; ces zones sont composées de chênaie, de pelouses, de cultures de céréales à paille (orge), de prairies temporaires et de prairies naturelles. Existent également des zones dépressionnaires de taille, forme et densité variables (dolines ou vallées sèches), aux sols plus profonds, parfois argileux, variablement caillouteux ; ces zones sont composées de prairies naturelles, de prairies temporaires ou de cultures assolées (luzerne, céréales d'hiver, maïs ou sorgho).

4) À l'ouest du département, la Bouriane jouxte la Dordogne. Cette région aux sols caillouteux, calcaires et superficiels se caractérise par des dépôts sidérolithiques (sables siliceux et argiles à graviers de couleur rouge, mais aussi lits de galets mêlés de quartz et concrétions ferromanganèses) et des altérites du Crétacé (argiles kaoliniques rougeâtres comprenant débris calcaires, silex et concrétions ferrugineuses ou ferromanganèses, sables quartzeux fins). Constitué de nombreux plateaux ondulés plus ou moins étroits (plateaux calcaires karstifiés), le paysage l'est aussi de vallées sèches, dolines, versants et collines à pentes fortes. Seules les dolines aux sols rouges argileux accueillent les cultures agricoles. Des pelouses sèches, peuplements de chênes pubescents, bois de châtaigniers et de résineux mais aussi des landes à Éricacées (le Frau à Lavercantière) ornent certains plateaux.

5) Au sud du département, on trouve le Quercy blanc ou Pays de Serres formé de plusieurs plateaux de calcaire lacustre très pauvre où les végétaux peinent à survivre; l'agriculture 
se concentre dans les petites vallées séparant ces plateaux.

Outre les vastes ensembles paysagers de ces petites régions, deux grandes rivières à l'origine d'importantes vallées traversent le département d'est en ouest :

- Le Lot prend sa source en Lozère, traverse des terrains acides du nord de l'Aveyron, puis les calcaires secondaires (Causses) du Lot. La vallée reste étroite sur ce parcours. En amont de Cahors, des falaises se dressent, nées des entailles infligées par le Lot aux calcaires durs du Jurassique. En aval, ce sont les calcaires marneux du Kimméridgien que le Lot entailla: les falaises sont plus rares et la vallée plus large ; les sols s'y composent d'alluvions et dépôts alluviaux.

- La Dordogne, au nord du Lot, n'y fait qu'une incursion d'une cinquantaine de kilomètres. Quittant les terrains acides du Primaire, elle entaille les calcaires du Secondaire. Près du lit majeur de la rivière, différentes alluvions sont disposées en terrasses basses, moyennes et supérieures ; galets, sables et limons composent des dépôts pliocènes. Grandes cultures, noyeraies et maraîchage forment la couverture très agricole des sols.

Présentation du groupe taxonomique des Anthophila

Le groupe considéré est celui des Hyménoptères Apiformes, autrement appelés Anthophiles, les abeilles au sens large, qui remonte à plus de 120 millions d'années. Apiformes et Sphéciformes constituent la superfamille des Apoidea. Un tableau du cadre systématique de classification des Hyménoptères Apoidea considérés dans l'article est présenté (figure 1). Indispensables à la pollinisation de nombreuses cultures, les Apiformes nidifient dans divers substrats : en majorité dans le sol, également dans des cavités naturelles (coquilles d'escargot, fissures ou fentes de rochers, tiges creuses, bois mort...) voire artificielles (trous d'aération de fenêtres, hôtels à insectes...). Quelques espèces sont sociales (les bourdons, l'abeille domestique, certains Halictidés), mais la plupart sont solitaires. La nomenclature utilisée pour désigner les taxons présentés dans cet article est celle de RASMONT et al. (2017), qui ont publié un catalogue des Anthophila de France, Belgique, Luxembourg et Suisse.

Les informations collectées sur les différents genres et espèces d'abeilles proviennent de plusieurs publications et sites Internet indiqués dans la bibliographie : Atlas Hymenoptera (www.atlashymenoptera.net/), IUCN (www. iucnredlist.org/), Observatoire des Abeilles (https://oabeilles.net). En France, 6 familles d'abeilles solitaires (présentées ci-après), 51 genres et 967 espèces ont été identifiés. Tous les chiffres indiqués dans cet article pour la faune de France sont basés sur TAXREF 13 (INPN, GARGONOMY et al. 2018).

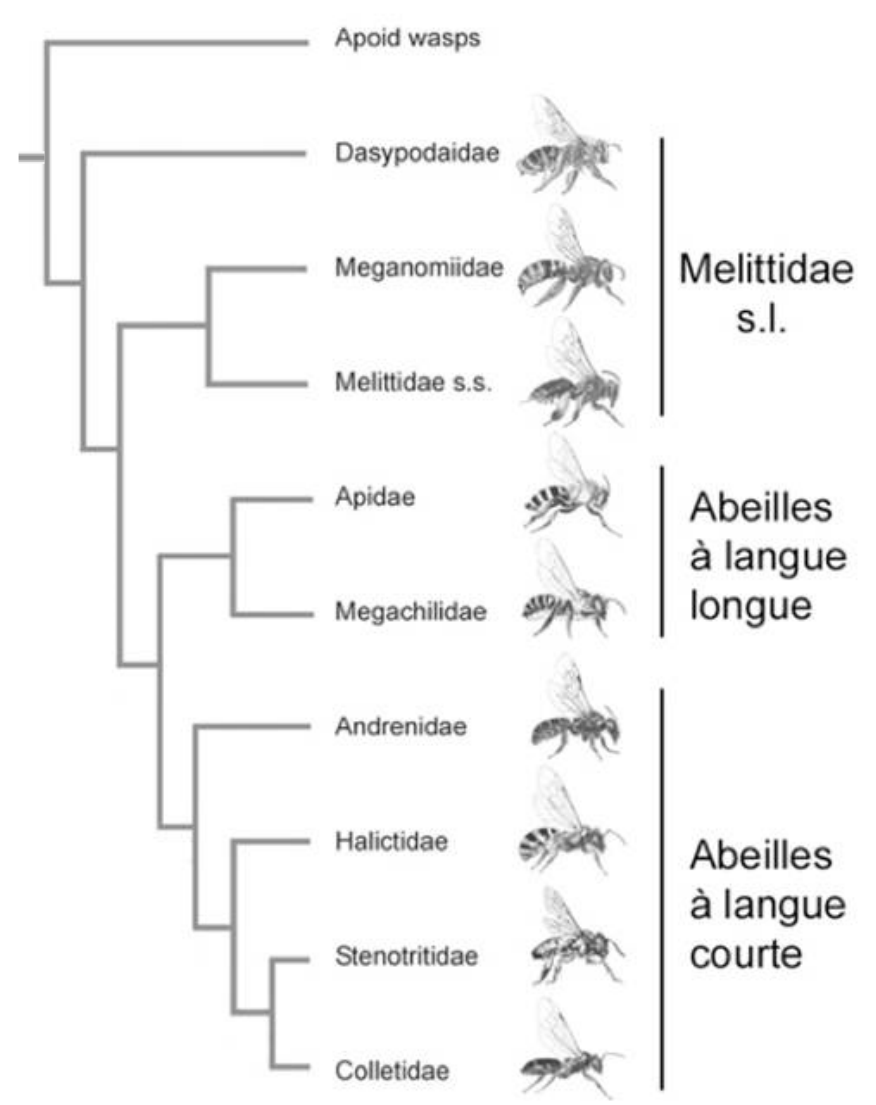

Figure 1. Classification des abeilles de DANFORTH et al. (2006a, b) redessinée par MICHEZ (2007).

\section{Matériel et Méthodes}

\section{$\underline{\text { Zone de prospections }}$}

L'ensemble des localités inventoriées de 1965 à nos jours est indiqué sur la carte II ci-après ; les noms de toutes les localités ou communes sont abrégés. Les observations présentées dans cet article ont été établies dans 73 communes du Lot ; sur les 7000 données réunies, quelques centaines 
sont antérieures à 2000. Les ronds blancs entourant des communes sur la carte signalent la capture d'espèces remarquables ou rares.

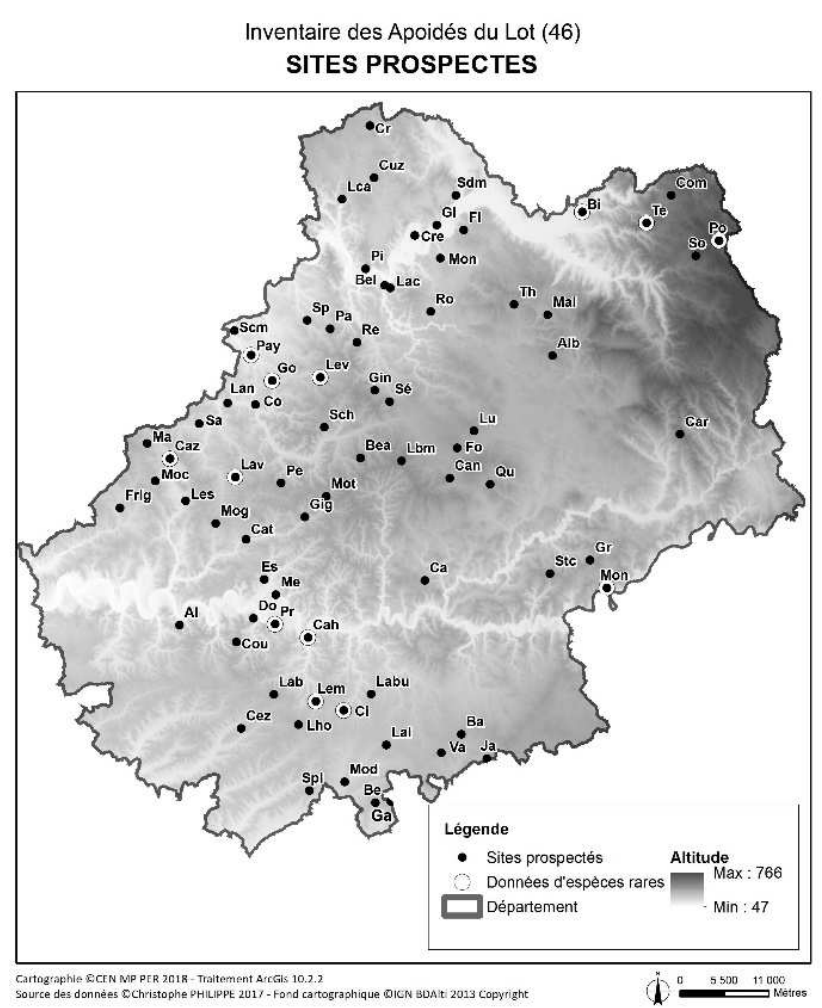

Carte II. Sites prospectés.

Al, Albas ; Alb, Albias ; Ba, Bach ; Bel, Belcastel ; Bea, Beaumat ; Be, Belfort-du-Quercy ; Bi, Biarssur-Cère ; Ca, Cabrerets ; Cah, Cahors ; Can, Caniac-du-Causse ; Car, Cardaillac ; Cat, Catus ; Cay, Caylus ; Caz, Cazals ; Ci, Cieurac ; Com, Comiac ; Co, Costeraste ; Cr, Cressenssac ; Cre, Creysse ; Cuz, Cuzance ; Do, Douelle ; Es, Espère; Fl, Floirac ; Fo, Fontanes-du-Causse ; Frlg, Frayssinet-le-Gélat ; Ga, Galdus ; Gig, Gigouzac ; Gin, Ginouillac ; Gl, Gluges ; Go, Gourdon ; Gr, Gréalou ; Ja, Jamblusse ; Lab, LabastideMarnhac ; Lbm, La Bastide-Murat ; Lan, L'Abbaye-Nouvelle ; Labu, Laburgade ; Lac, Lacave ; Lal, Lalbenque ; Lca, La ChapelleAuzac ; Lav, Lavercantière ; Lem, Le Montat ; Lev, Le Vigan ; Les, Les Arques ; Lho, L'Hospitalet ; Lu, Lunegarde ; Ma, Marminiac ; Mal, Mayrinhac-Lentour ; Me, Mercuès ; Mot, Montamel ; Mon, Montbrun ; Moc, Montcléra ; Mod, Montdoumerc ; Mov, Montvalent ; Mog, Montgesty ; Par, Payrac ; Pay, Payrignac ; Pe, Peyrilles ; Pi, Pinsac ; Pr, Pradines ; Qu, Quissac ; Re, Reilhaguet ; Ro, Rocamadour ; Sa, Salviac ; Sé, Séniergues ; Sp, Serres-Payrac ; Stc, SaintChels ; Scm, Saint-Cirq-Madelon ; Sdm, SaintDenis-lès-Martel ; Spl, Saint-Paul-de-
Loubressac ; So, Sousceyrac ; Te, Teyssieu ; Th, Thégra ; Va, Vaylats.

\section{Validation des données}

Les données antérieures à 2000 ayant permis l'établissement de cette liste des abeilles sauvages $\mathrm{du}$ département $\mathrm{du}$ Lot proviennent des prospections effectuées par Gérard CANOU et Marc TussAC. Les données postérieures à 2000 résultent des prospections réalisées par l'auteur en fonction de ses disponibilités ainsi que des données transmises par des spécialistes, mais aussi des captures réalisées par le Conservatoire des espaces naturels de Midi-Pyrénées (Samuel DANFLOUS). La plupart des spécimens ont été capturés à vue au filet avec cependant quelques captures effectuées par le truchement de coupelles colorées ou de tentes MALAISE. Les identifications ont été dans un premier temps réalisées par l'auteur puis vérifiées et validées par différents spécialistes français ou étrangers : Gilles MAHÉ pour les Bourdons, Gérard LE GoFF et David GENOUD pour les Anthophores, Gérard LE GOFF pour les Xylocopinae, Michael KUHLMANN et David Genoud pour les Colletes, David GENOUD pour les Melittidae, Matthieu AUBERT pour les Megachilidae, Alain PAULY pour les Halictidae, David GENOUD pour les Andrenidae, Stephan RISH et MATTHIEU Aubert pour les Eucerini, Éric DUFRÊNE pour les abeilles-coucous, Serge GADOUM et Matthieu AUBERT pour les Hylaeus. La totalité des spécimens capturés se trouve chez l'auteur, à l'exception de quelques spécimens conservés dans les collections des spécialistes cités ci-dessus.

\section{Résultats et discussion}

\section{$\underline{\text { Bilan global de l'inventaire }}$}

Comme indiqué précédemment, les 967 espèces d'Anthophila répertoriées en France se répartissent comme suit : $30 \%$ d'Apidae, $21 \%$ de Megachilidae, $20 \quad \%$ d'Andrenidae, $19 \%$ d'Halictidae, $8 \%$ de Colletidae et $1,5 \%$ de Melittidae (figure 3b). Le Lot comprend 398 espèces réparties en 6 familles (figures 2 et $3 \mathrm{~b}$ ) données dans l'ordre croissant de leur richesse en espèces : Apidae (110 espèces, soit $28 \%$ du total des espèces), Andrenidae (92 espèces, soit $23 \%$ ), Halictidae (82 espèces, soit 20,5 \%), Megachilidae (80 espèces, soit $20 \%$ ), Colletidae (27 espèces, soit $7 \%$ ) et Melittidae ( 7 espèces, soit $2 \%$ ). 


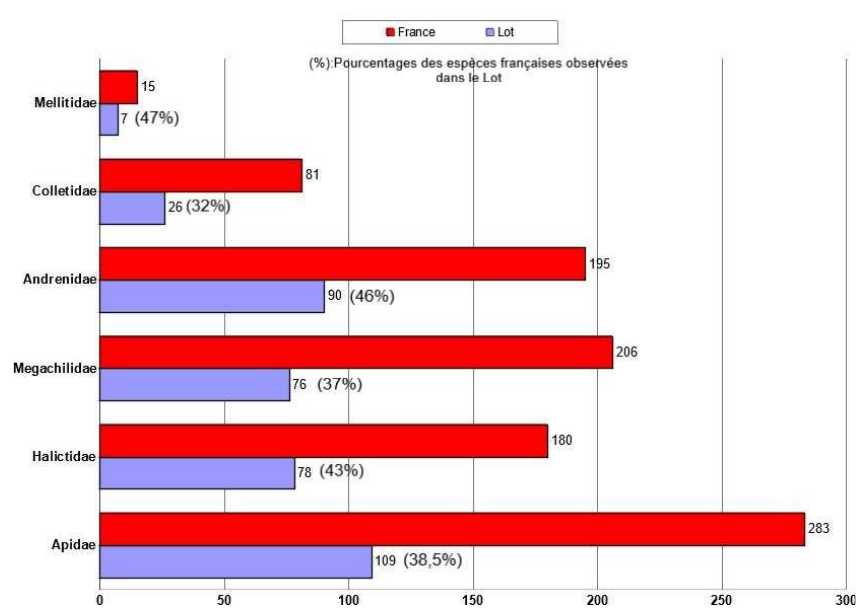

Figure 2. Comparaison du nombre d'espèces par famille entre le Lot et la France

La distribution des espèces par famille (figure 3b) est très proche de celle observée au niveau national (figure 3a). En effet, les Apidae sont présents en France à hauteur de $29 \%$ et de $28 \%$ dans le Lot ; les proportions d'Andrenidae, Megachilidae et Halictidae sont, dans les deux cas, proches de $20 \% ; 7-8 \%$ de Colletidae et $2 \%$ de Melittidae sont recensés dans l'Hexagone et dans le Lot.

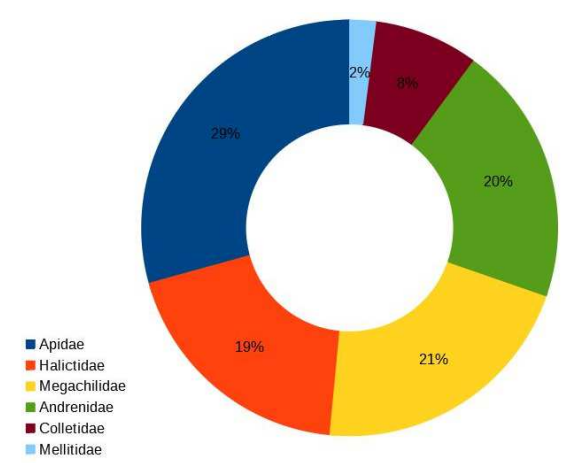

Figure 3a. Répartition des espèces par famille pour la France en (\%)

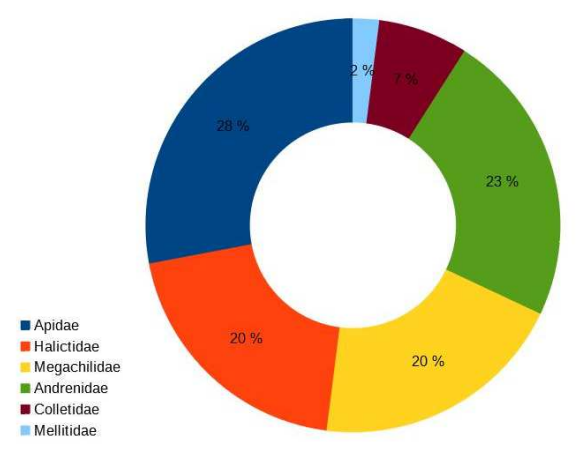

Figure 3b. Répartition des espèces par famille pour le Lot en (\%)

Les 398 espèces comptabilisées dans ce département représentent environ $41 \%$ de la totalité des espèces dénombrées en France (967).
Les Apidae répertoriés dans le Lot représentent 38,5 \% des Apidae recensés en France ; les Halictidae, $43 \%$; les Megachilidae, $37 \%$; les Andrenidae, $46 \%$; les Colletidae, $32 \%$ et les Melittidae, $47 \%$ (figure 2). Cette différence de pourcentage entre les familles est probablement due à une sous-prospection de certaines zones comme le Nord, le Nord-Est (Ségala) et le SudOuest (Quercy blanc).

Quatorze espèces parmi les 398 recensées n'ont pas été revues depuis 1965 ainsi que 4 espèces depuis 1999. D'autres taxons supplémentaires en cours de validation et/ou à déterminer appartenant principalement aux familles des Halictidae et Colletidae n'ont pas été intégrés au présent inventaire - ce qui peut expliquer la différence de pourcentage entre espèces pour ces dernières familles par rapport à la faune française.

Si l'on compare la présente liste avec l'étude publiée pour le département voisin de l'Aveyron (BALITEAU et al., 2013) - laquelle recense 199 espèces (67 Apidae, 47 Halictidae, 38 Megachilidae, 40 Andrenidae, 7 Colletidae et 2 Melittidae), soit environ $21 \%$ des espèces d'abeilles comptabilisées en France - on constate qu'elle est plus riche en espèces, bien que 72 communes seulement aient été visitées (1 180 stations environ l'ont été dans l'Aveyron, le nombre total de communes prospectées n'étant pas connu). Cette différence en termes de nombre d'espèces comptabilisées dans ces deux études peut provenir du travail réalisé pour approfondir les connaissances de la phénologie et de la biologie des espèces d'Anthophila - connaissances qui renforcent l'efficacité au niveau des prospections sur le terrain et permettent de mieux évaluer la richesse intrinsèque de la faune lotoise.

La technique, principalement mise en œuvre, de capture au filet (collecte à vue) pourrait expliquer les faibles effectifs de Colletidae et d'Halictidae dénombrés. L'utilisation complémentaire de pièges du type tente Malaise ou coupelles colorées pourrait permettre d'enrichir cet inventaire faunistique. À l'avenir, certaines espèces montagnardes appartenant aux autres familles d'abeilles pourraient être capturées en augmentant l'effort de prospection dans le Ségala lotois, le causse de Martel et le Quercy blanc ainsi que dans différents biotopes classés en ZNIEFF et en zones Natura 2000. 
Biologie et écologie des abeilles du Lot

La socialité des abeilles, leurs modes de nidification (emplacements des nids et matériaux de construction), les ressources alimentaires qu'elles exploitent et le climat des aires géographiques où elles évoluent constituent des caractéristiques écologiques importantes. De nombreux traits morphologiques et l'occurrence de ces abeilles solitaires sont associés à ces caractéristiques écologiques. Un tableau, directement publié sur le site de l'Observatoire des Abeilles ou celui de la revue Osmia (PHILIPPE 2020), résume ces informations et propose, pour chaque espèce, la catégorie dans laquelle la Liste rouge européenne (IUCN) l'a classée ainsi que sa valeur patrimoniale au niveau départemental.

Parmi les 398 espèces recensées dans le Lot, 103 sont des taxons " cleptoparasites" répartis sur 12 genres (PHILIPPE 2020) ; ils représentent $26 \%$ du total des espèces répertoriées dans ce département contre $22 \%$ du total des espèces listées en France. Ces abeilles-coucous pondent leurs œufs dans le nid d'autres espèces d'abeilles après que ces dernières ont amassé des provisions dans les cellules larvaires. Ne récoltant ni pollen ni nectar pour leur progéniture, elles sont dépendantes des espèces " hôtes "; certaines d'entre elles semblent exclusivement liées à une seule espèce d'abeillehôte. Dans le Lot, 4 familles d'abeilles (Apidae, Colletidae, Halictidae, Megachilidae) sur les 6 existantes comprennent des espèces cleptoparasites, capturées essentiellement à vue au filet car les pièges colorés ne les attirent pas ou peu.

Chez les 295 autres espèces d'abeilles non cleptoparasites (74 \% des espèces dénombrées dans le Lot), la stratégie de recherche de nourriture (pollen) est différente. Dites généralistes ou polylectiques, certaines d'entre elles se nourrissent d'un large éventail d'espèces végétales appartenant à divers genres et familles. Spécialisées, d'autres espèces limitent leurs visites à des taxons végétaux bien déterminés. Trois niveaux de lectisme ont été définis : $1^{\circ}$ le monolectisme (prélèvement de pollen sur une seule espèce de plante-hôte) ; $2^{\circ}$ l'oligolectisme (collecte pollinique au sein d'une famille de plantes-hôtes) ; $3^{\circ}$ le polylectisme au sens strict (pollen prélevé sur plus d'une famille de planteshôtes) ou polylectisme avec une préférence marquée pour certains taxons végétaux (mésolectisme - CANE \& SIPES 2006 et modifié par MÜLLER \& KUHLMANN 2008). Comme déjà signalé, ces choix floraux ne concernent que les femelles qui achalandent elles-mêmes leurs cellules larvaires en nourriture. Dans cette liste des abeilles du Lot (PHILIPPE 2020), 189 espèces (soit $47 \%$ du total des espèces dénombrées dans ce département) appartenant à 16 genres (47 espèces d'Andrena, 10 Anthophora, 13 Bombus, 5 Ceratina, 1 Apis, 4 Eucera, 3 Xylocopa, 1 Colletes, 15 Hylaeus, 16 Halictus, 37 Lasioglossum, 1 Nomiapis, 8 Anthidium, 6 Hoplitis, 8 Osmia, 14 Megachile) sont polylectiques au sens strict ou avec une préférence marquée ; et 101 espèces (soit $25 \%$ du total des espèces recensées dans le Lot) réparties en 16 genres (43 espèces d'Andrena, 2 Panurgus, 3 Anthophora, 5 Tetraloniella, 7 Colletes, 2 Hylaeus, 2 Lasioglossum, 1 Rophites, 2 Trachusa, 6 Chelostoma, 2 Heriades, 3 Hoplitis, 12 Osmia, 2 Lithurgus, 2 Megachile, 4 Melitta, 1 Macropis, 2 Dasypoda) sont oligolectiques. Mis à part le lectisme des espèces cleptoparasites non pris en compte, celui d'une espèce en particulier (Hylaeus sinuatus) est inconnu. L'appareil de récolte du pollen varie d'une famille d'abeilles à l'autre : corbicules sur les pattes postérieures des Apis et Bombus (Apidae) ou bien brosse ventrale des Megachilidae.

La récolte du nectar s'effectue quant à elle sur un plus large éventail d'espèces végétales ; les morphologies de la fleur, mais aussi de l'abeille (taille du corps, longueur et structure de la langue, notamment les palpes labiaux et maxillaires) l'influencent. Les abeilles à langue longue (Apidae, Megachilidae) sont capables de recueillir le nectar des fleurs à longue corolle tubulaire tandis que les abeilles à langue courte (Andrenidae, Colletidae, Halictidae et Melittidae) ne butinent de nectar que sur des fleurs à tube corollaire court ou dont la corolle est courte et largement ouverte. En outre, la distance maximale parcourue entre les ressources alimentaires et les sites de nidification varie d'une espèce à l'autre (d'une centaine de mètres pour les Lasioglossum à plusieurs kilomètres pour les Bombus par exemple).

\section{Modes de nidification}

Ont été comptabilisées 198 espèces nichant dans le sol (sur un total de 398 espèces, soit $50 \%$ d'espèces terricoles) et 92 espèces vivant dans des 
cavités (superficielles ou naturelles) ou au-dessus $\mathrm{du}$ sol (soit $23 \%$ du total des espèces lotoises recensées). Le mode de nidification de 5 espèces est à ce jour inconnu (Andrena nitidula, Chelostoma emarginatum, Chelostoma foveolatum, Hoplitis antigae, Megachile burdigalensis). Pour les abeilles creusant des terriers comme les Andrenidae, la texture du sol et l'exposition au soleil du site de nidification sont d'importants critères ; citons par exemple Andrena vaga, qui nidifie en bourgades dans un sol plutôt sableux en bordure de rivière, ou Panurgus calcaratus qu'on trouve plutôt dans le Ségala sur sols sableux en bordure de chemins agricoles au sein de zones humides. Les Colletidae et Halictidae sont aussi des abeilles terricoles (Colletes hederae ou Halictus scabiosae par exemple).

Certaines espèces non-terricoles sont xylocoles ou rubicoles : elles creusent des galeries dans le bois mort ou des tiges végétales (genres Hylaeus, Xylocopa ou Ceratina : il n'est pas rare de trouver en hiver des cératines regroupées au sein des tiges sèches de Rubus où elles nidifient). Certaines autres espèces nichent dans des cavités préexistantes : nids anciens d'Hyménoptères, fissures de rochers, coquilles d'escargots (telles certaines osmies hélicicoles : $O$. andrenoides, $O$. aurulenta, O. bicolor, O. versicolor, O. viridana que l'on trouve plutôt dans des milieux thermophiles comme les pelouses sèches).

Selon l'espèce, différents matériaux de construction peuvent être utilisés pour fabriquer les nids ou tapisser les cellules du couvain : morceaux de feuilles mastiquées, pétales de fleurs, terre, petits cailloux, etc. (MICHENER 2007 ; MÜlleR 2011 ; VEREECKEN 2017). Par exemple, Osmia (Tergosmia) tergestensis DUCKE, 1897 une espèce peu observée dans le Lot - fait son nid dans des cavités ou fissures rocheuses ou même dans des touffes d'herbes denses (truffières en friche). Elle construit ses cellules individuellement ou par petits groupes (comprenant 5 cellules ou plus proches les unes des autres). Celles qui ne sont pas collées au substrat se composent de 3 strates: les couches extérieure et intérieure sont faites de gros morceaux de pétales de fleurs (par exemple de Geranium, Helianthemum, Hieracium ou Ononis) et la couche centrale est construite de boue mélangée de petits cailloux. Dans les cavités étroites, l'espace situé devant les cellules est parfois rempli de miettes de terre sur une longueur pouvant aller jusqu'à $0,5 \mathrm{~cm}$ (AMIET et al. 2004 ; BANASZAK \& ROMASENKO 2001 ; BENOIST 1931 ; FERTON 1897 ; MÜLLER et al. 1997 ; ROZEN et al. 2010).

\section{$\underline{\text { Socialité }}$}

Le degré de socialité des abeilles est très variable et complexe (MichenER 2007). La majorité des espèces répertoriées dans le Lot (254 espèces sur un total de 398, soit $64 \%$ des abeilles lotoises recensées) est solitaire. Les 37 espèces sociales (ou eusociales, environ $10 \%$ du total des espèces lotoises dénombrées) ont des interactions et des modes de vie plus complexes. Au début du cycle de vie des espèces sociales, des reines fécondées construisent un nid dans un endroit choisi avant d'y élever un couvain d'ouvrières qui entretiendront le nid et collecteront les ressources alimentaires. Chez certaines espèces, il n'y a aucune différence entre la reine et les ouvrières vivant dans le même nid. Dans les ruches de l'abeille domestique en revanche, la seule reine fécondée existante est dissociable des ouvrières (comme chez les bourdons). Chez les Halictidae, une socialité bien développée existe (eusocialité), plus ou moins importante en fonction des espèces, lesquelles présentent une tendance à se regrouper en bourgades pour élaborer leurs nids (telles qu'Halictus scabiosae et Lasioglossum spp. par exemple).

Compte tenu de la variété des modes de nidification et des ressources alimentaires de ces Anthophila, il importe de conserver dans le paysage une mosaïque d'habitats permettant le maintien de microsites favorables à la nidification sur une superficie de quelques centaines ou dizaines de mètres. Les milieux du type "openfields» ou les habitats particuliers comme les marais, les prairies trop humides ou modifiées (épandage, densification des graminées, surpâturage) ou les milieux boisés sont très peu favorables aux abeilles, à l'exception de quelques espèces très spécialisées. En revanche, lorsqu'il est très local, le sur-piétinement (passage d'animaux, chemins agricoles) ou le surpâturage peuvent favoriser la création d'espaces de nidification ( $\mathrm{sol} \mathrm{nu}$ ) très prisés des abeilles et de leurs parasites (abeilles-coucous), mais aussi d'autres insectes comme les Diptères, Coléoptères et Hyménoptères. 
Évaluation du statut patrimonial des espèces au niveau départemental

L'étude des Anthophila du Lot a 2 objectifs principaux :

$\rightarrow$ améliorer la connaissance de ces pollinisateurs à l'échelle départementale ;

$\rightarrow$ améliorer les connaissances du grand public et des naturalistes sur ce groupe d'insectes grâce à une plateforme de données naturalistes consultable sur Internet développée par le CEN (Conservatoire des espaces naturels) MidiPyrénées en lien avec le SINP (Système d'information sur la nature et les paysages).

Ces 2 objectifs concourent au PRA (Plan régional d'actions) pollinisateurs piloté par l'OPIE (Office pour les insectes et leur environnement) Occitanie et la DREAL (Direction régionale de l'environnement, de l'aménagement et du logement) Occitanie. Voir le site Internet dédié : https://pollinisateurs.pnaopie.fr/plan-national.

\section{Protocole d'évaluation}

Les Listes rouges classifient les espèces en fonction de leur risque d'extinction dans les zones géographiques considérées. Les catégories et critères de la Liste rouge de l'UICN fournissent un cadre explicite et objectif à ces évaluations (UICN 2018). À l'échelle nationale, les espèces sont classées en 11 catégories (figure 4) sur la base d'un ensemble de critères quantitatifs liés à la taille, à la structure, et à l'aire géographique des populations considérées (UICN 2018 ; NIETO et al.). Les espèces menacées sont classées en 3 catégories : Vulnérable (VU), Endangered (EN) et Critical Endangered (CR). Un taxon est considéré comme Quasi menacé (NT) lorsqu'après évaluation, il ne remplit pas les conditions exigées par les 3 catégories susmentionnées, mais s'avère proche de la qualification ou susceptible de se qualifier dans un avenir proche. Les espèces qui ne sont pas menacées entrent dans la catégorie Préoccupation mineure (LC). La catégorie DD (Data Deficient) regroupe toutes les espèces pour lesquelles le manque de données ne permet pas d'effectuer une évaluation.

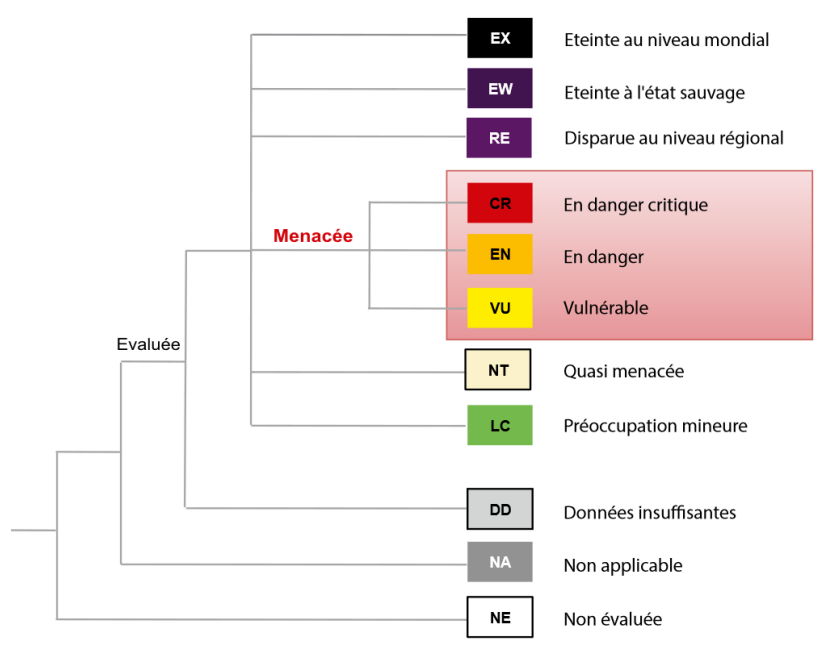

Figure 4. Typologie des statuts conservatoires de I'Union internationale pour la

Conversation de la Nature (UICN, 2018).

Les acronymes standards correspondent à la dénomination des catégories en anglais et sont utilisés tels quels dans toutes les langues : $\mathrm{EX}=$ Extinct, $\mathrm{EW}=$ Extinct in the wild, $\mathrm{RE}=$ Regionally extinct, $\mathrm{CR}=$ Critically endangered, $\mathrm{EN}=$ Endangered, $\mathrm{VU}=$ Vulnerable, NT = Near threatened, $\mathrm{LC}=$ Least concern, DD = Data deficient, NA = Not applicable, NE = Not evaluated .

\section{Protocole adopté afin d'évaluer la valeur patrimoniale des abeilles du département du Lot}

Compte tenu du manque important de données d'inventaires disponibles, apprécier la valeur patrimoniale des abeilles lotoises s'avère difficile. Notre analyse s'appuie essentiellement sur différents documents comme les Listes rouges publiées dans plusieurs pays européens (site Internet de l'IUCN), les données du site Atlas Hymenoptera (http://zoologie.umh.ac.be/ hymenoptera) pour différents genres (Bombus, Anthophora, Eucera, Melitta, Megachile, Halictus, Lasioglossum), mais aussi du site FlorAbeilles (www.florabeilles.org) mis au point par l'équipe de Bernard VAISSIÈRE de l'INRA d'Avignon. Au niveau départemental, l'auteur utilise sa base de données pour évaluer le statut de conservation de chaque espèce présentée.

L'échelle de temps à laquelle l'UICN recourt afin d'évaluer la variation de taille d'une population est de 10 ans (ou de 3 générations). Dans le cadre de cette liste préliminaire, 3 périodes ont été comparées : avant 1965, de 1965 à 1999 et de 2000 à 2018. Initialement, l'objectif de la définition de ces 3 périodes était d'identifier vers quel état de conservation tendent les espèces lotoises listées mais, en raison du manque de données pour les 2 premières périodes, le protocole IUCN s'avère non 
applicable ; l'analyse portera donc sur l'ensemble des observations, toutes périodes confondues.

Comparer différentes périodes implique de tenir compte des changements dans le nombre et le comportement des observateurs. Ces changements s'expriment par des différences dans les méthodes d'échantillonnage (par exemple, les stratégies d'échantillonnage axées ou non sur les points chauds et/ou les espèces facilement reconnaissables, les zones et fréquences d'échantillonnage), le nombre d'enregistrements des données plus récentes et les objectifs de l'échantillonnage (par exemple, inventorier un lieu privilégié, comparer les données récentes avec les données anciennes relevées au même endroit, répondre à des programmes de recherche, rechercher des espèces rares à des fins de conservation...). L'appréciation de la valeur patrimoniale d'une espèce répertoriée dans le Lot est donc soumise à ces critères. Toutes périodes confondues, une espèce qui n'a été capturée que dans 2 stations maximum est classée " + " (espèce peu abondante) ; une espèce capturée dans 2 à 10 stations est classée "++» (espèce d'abondance moyenne) ; au-delà de 10 stations, elle entre dans la catégorie « +++ » (espèce abondante).

\section{Résultats de l'évaluation}

L'analyse des données permet d'affirmer que, tous genres confondus, 150 espèces sont classées «+ peu abondantes", 164 espèces «++ d'abondance moyenne" et 84 espèces " +++ abondantes". Parmi les 150 espèces peu abondantes, 43 sont oligolectiques (spécialistes), 57 sont polylectiques et 49 sont des abeilles-coucous (cleptoparasites). Certaines espèces oligolectiques sont des spécialistes de biotopes précis. Par exemple, certains andrènes comme $A$. apicata, $A$. batava, $A$. praecox, $A$. vaga ou $A$. ventralis sont strictement inféodées aux saules (Salix spp.) pour le pollen et le sont donc aussi aux biotopes de vallées fluviales (qu'il convient de préserver).

En annexe, seules 5 catégories de classement UICN ont été conservées : EN : En voie d'extinction ; VU : Vulnérable ; NT : Quasimenacé ; LC : Préoccupation mineure ; DD : Données insuffisantes. Le tableau de synthèse est disponible en ligne (occurrences et traits de vie des abeilles solitaires) (PHILIPPE 2020). On dénombre pour le département du Lot : 2 espèces classées EN (En danger), 1 espèce en VU (Vulnérable), 25 espèces en NT (Quasi-menacées), 277 espèces en LC (Préoccupation mineure) et 93 espèces en DD (Manque de données). Si l’on regroupe les espèces classées dans les catégories "En danger", "Vulnérable » et "Quasi-menacé », on obtient un total de 28 espèces au regard des données existantes : Trachusa byssina (EN), Lasioglossum laeve (EN), Colletes fodiens (VU) ; les espèces suivantes sont classées NT : Andrena gallica, Andrena hattorfiana, Andrena ovatula, Epeolus cruciger, Nomada armata, Nomada errans, Nomada mutica, Nomada villosa, Colletes succinctus, Halictus quadricinctus, Lasioglossum angusticeps, Lasioglossum clypeare, Lasioglossum laevigatum, Lasioglossum majus, Lasioglossum sexnotatum, Lasioglossum xanthopus, Rophites quinquespinosus, Sphecodes croaticus, Sphecodes hyalinatus, Sphecodes majalis, Sphecodes rubicundus, Sphecodes schenckii, Sphecodes spinulosus, Melitta tricincta, Dasypoda argentata. Parmi ces 28 espèces, 50 \% sont des Halictidae, 18 $\%$ des Apidae, $11 \%$ des Andrenidae, $3 \%$ des Colletidae et $7 \%$ des Melittidae.

Si l'on recourt aux critères qui permettent d'apprécier la valeur patrimoniale des espèces dénombrées dans le Lot ainsi qu'aux critères du classement UICN s'appliquant aux mêmes espèces, des différences apparaissent. Ainsi, d'après notre liste (PHILIPPE 2020), parmi les 28 espèces classées $\mathrm{EN}, \mathrm{VU}$ et $\mathrm{NT}, 3$ s'avèrent abondantes dans le département : Andrena ovatula, Halictus quadricinctus, Sphecodes majalis.

Parmi les 93 espèces classées dans la catégorie DD, 43 apparaissent comme peu abondantes (23 Andrenidae, 10 Apidae, 3 Colletidae, 1 Halictidae, 6 Megachilidae), 33 sont d'abondance moyenne (21 Andrenidae, 3 Apidae, 3 Halictidae, 6 Megachilidae) et 17 sont abondantes (11 Andrenidae, 2 Apidae, 4 Megachilidae).

Si l'on se base sur le classement de l'UICN, des espèces appartenant à plusieurs sous-familles ne sont pas considérées comme menacées (Panurginae, Xylocopinae, Anthophorinae, Bombinae, Eucerinae, Melectinae, Hylaeinae, Megachilinae, Osminae, Lithurginae). Pourtant, la première évaluation patrimoniale de l'abondance des espèces réalisée au niveau départemental fait le constat que, parmi les 277 espèces classées dans la catégorie LC, 93 
apparaissent comme peu abondantes dans le Lot (11 Andrenidae, 31 Apidae, 11 Colletidae, 12 Halictidae, 26 Megachilidae, 2 Melittidae (PHILIPPE 2020).

\section{Présentation de quelques espèces remarquables}

- Andrena bicolorata (Rossi, 1790) : jolie espèce à poils blancs semblant oligolectique sur différentes Brassicacées. Découverte seulement en 2017 dans le Lot, son aire de répartition actuelle se situe sur le pourtour méditerranéen.

- Andrena congruens (SCHMIEDEKNECHT, 1882) : cette espèce orophile des biotopes humides est mésolectique (préférence pour les Apiacées) et bivoltine (2 générations par an). Classée LC par l'IUCN, elle n'a été vue dans le Lot que rarement depuis 1980. Elle a été redécouverte en 2017 dans le Frau de Lavercantière.

- Andrena limata (SMITH, 1853) : cette espèce polylectique, qui n'a pas été revue depuis 1956 dans le Lot, est considérée comme disparue en Ile-de-France.

- Andrena niveata (FRIESE, 1887) : cette petite espèce du sous-genre Micrandrena qui habite divers habitats est spécialisée sur les Brassicacées pour le pollen. Elle n'a pas été revue dans le Lot depuis 1955.

- Andrena potentillae (PANZER, 1809) : cette petite andrène rouge oligolectique a été découverte en 2016 à Gréalou sur potentille ce qui est étonnant, car elle évolue habituellement dans le nord du Massif Central et l'est de la France.

- Anthophora femorata (OLIVIER, 1789) : cette très belle abeille au vol très rapide est oligolectique sur Echium (Boraginacées) ; aussi est-elle à rechercher sur cette plante en juillet-août. Nul ne l'a revue depuis 1949 dans le Lot.

- Epeolus cruciger (PANZER, 1799) : cette abeille cleptoparasite classée NT par l'IUCN et peu abondante dans le Lot parasite les nids des abeilles du genre Colletes et notamment, Colletes succinctus, espèce liée aux landes à bruyère (Calluna vulgaris), biotopes très menacés dans toute l'Europe.

- Melecta luctuosa (SCOPOLI, 1770) : cette abeille-coucou - qui parasite des anthophores du sous-genre Pyganthophora comme Anthophora retusa et Anthophora aestivalis est devenue rare en France, mais aussi dans le Lot, en raison de la régression de ses hôtes.

- Eucera caspica (MORAWITZ, 1873) : cette abeille méditerranéenne a été découverte en 2018 à Rocamadour sur des Fabacées (première donnée).

- Nomada armata (HERRICH-SCHÄFFER, 1839) : cette Nomada, qui parasite Andrena hattorfiana, est en régression car l'intensification des pratiques agricoles a réduit la surface de l'habitat approprié et des ressources florales de son hôte. Elle est classée Quasi-menacée (NT) dans la Liste rouge européenne.

- Nomada blepharipes (SCHMIEDEKNECHT, 1882) : non revue depuis 1962 , cette abeillecoucou a probablement pour hôte Andrena hypopolia (KOCOUREK 1966, AMIET et al. 2007), peut-être Andrena cordialis.

- Nomada bispinosa (MOCSARY, 1883) : C'est une espèce peu commune, à l'hôte incertain (Lasioglossum spp.). Elle n'a pas été revue dans le Lot depuis 1954 !

- Nomada errans (LEPELETIER, 1841) : redécouverte en 2019 dans le Lot, cette abeillecoucou classée NT a été capturée pour la dernière fois en 1965. Il y a peu de données pour la France. Oligolectique sur les Apiacées et observable dans les biotopes xérophiles en juillet-août, elle parasite Andrena pallitarsis (CELARY 1995).

- Nomada linsenmaieri (SCHWARZ, 1974) : capturée pour la première fois en 2017 dans une seule station, son hôte reste inconnu.

- Nomada mutica (MoRAWITZ, 1872) : peu abondante dans le Lot, cette abeille-coucou parasite Andrena ferox (STOECKHERT 1933, KoCOUREK 1966, SMIT 2006). Elle est classée 
Quasi-menacée (NT) à cause des changements dans les pratiques agricoles.

- Nomada villosa (Thomson, 1870) : peu abondante dans le Lot (quelques stations et captures étalées dans le temps), son hôte est Andrena lathyri, oligolectique sur Fabacées, espèce beaucoup plus courante. Cette Nomada est sur la Liste rouge européenne et classée Quasi-menacée (NT).

- Colletes hylaeiformis (EVERSMANN, 1852): la dernière donnée date de 1957. Cette abeille à langue bifide est oligolectique sur Apiacées (Eryngium). Elle est à rechercher en juilletaoût.

- Lasioglossum majus (NYLANDER, 1852) : cette espèce d'assez grande taille pour le genre est polylectique. Elle n'a été trouvée que dans trois communes du Lot. Peu abondante dans le département, elle est considérée comme Quasi-menacée (NT) sur la Liste rouge européenne.

- Lasioglossum medinai (VACHAL, 1895) : séparée depuis peu d'avec $L$. villosulum (Kirby, 1802) (PAUlY et al. 2019), cette nouvelle espèce, non encore classée au niveau européen, est peu abondante dans le Lot.

- Lasioglossum sexnotatum (KIRBY, 1802) : classée Quasi-menacée (NT) et peu abondante dans le Lot, cette abeille solitaire de taille moyenne (et de grande taille à l'échelle du genre Lasioglossum) a été capturée récemment dans l'est du Lot, là où des zones de bocage existent encore.

- Sphecodes rubicundus (VON HAGENS, 1875): cette abeille-coucou qui parasite Andrena labialis (selon SowA \& MosTowsKA 1978 et BLÜTHGEN 1934) n'a été trouvée que dans 4 stations ; elle reste donc peu abondante au niveau départemental. Elle est classée Quasi-menacée (NT) selon la Liste rouge européenne.

- Chelostoma foveolatum (MORAWITZ, 1868) : Cette petite chélostome, qui s'avère oligolectique sur Campanulacées (AMIET et al. 2004, SEDIVy et al. 2008) et affectionne les milieux thermophiles, est peu abondante dans le Lot. Son mode de nidification est inconnu.
- Osmia xanthomelana (KIRBY, 1802): Cette osmie, qui niche dans la litière forestière, est peu abondante dans le Lot (observée une seule fois). Oligolectique sur les Fabacées notamment, elle a une préférence pour Lotus sp.

- Melitta nigricans (ALFKEN, 1905) : Peu abondante dans le Lot, cette abeille terricole est l'une des 4 espèces de Melitta qui s'y trouve. Elle est hyper spécialisée sur la salicaire (Lythrum salicaria), une plante liée aux zones humides et plus particulièrement aux mégaphorbiaies.

\section{Conclusion}

Riche de ses 398 espèces recensées, cette liste préliminaire des Hyménoptères Anthophila du Lot marque une étape dans la connaissance départementale de ce groupe et illustre la grande diversité des abeilles sauvages de ce territoire qui, par exemple, abrite aussi bien des taxons montagnards ou septentrionaux (Bombus subterraneus, Andrena congruens, Andrena vaga, Osmia xanthomelana) que des taxons strictement méditerranéens (Andrena granulosa, Anthophora femorata). Cette liste est cependant encore loin d'être exhaustive. Ainsi, un certain nombre d'espèces (appartenant notamment aux genres Hylaeus, Colletes et Nomada) n'y ont-elles pas encore été intégrées, car elles attendent la validation des spécialistes. Par ailleurs, des observations encore inédites sont probablement disponibles dans certaines collections ou bases de données à l'étranger (telles celles de Bombus pomorum par exemple), le département étant visité par de nombreux entomologistes amateurs ou professionnels. Enfin, des prospections plus ciblées sur les zones encore peu explorées du Ségala devraient permettre d'enrichir encore la liste d'espèces montagnardes.

Pour approfondir ce travail, il conviendrait à l'avenir de poursuivre les captures de spécimens en couplant les méthodes de collecte (captures à vue et méthodes normalisées) et ce, avec l'aide d'un groupe plus important de récolteurs afin d'augmenter le nombre d'espèces inventoriées, préciser la répartition de certaines espèces dans le département et y confirmer l'absence ou la rareté d'espèces communes dans d'autres régions. 

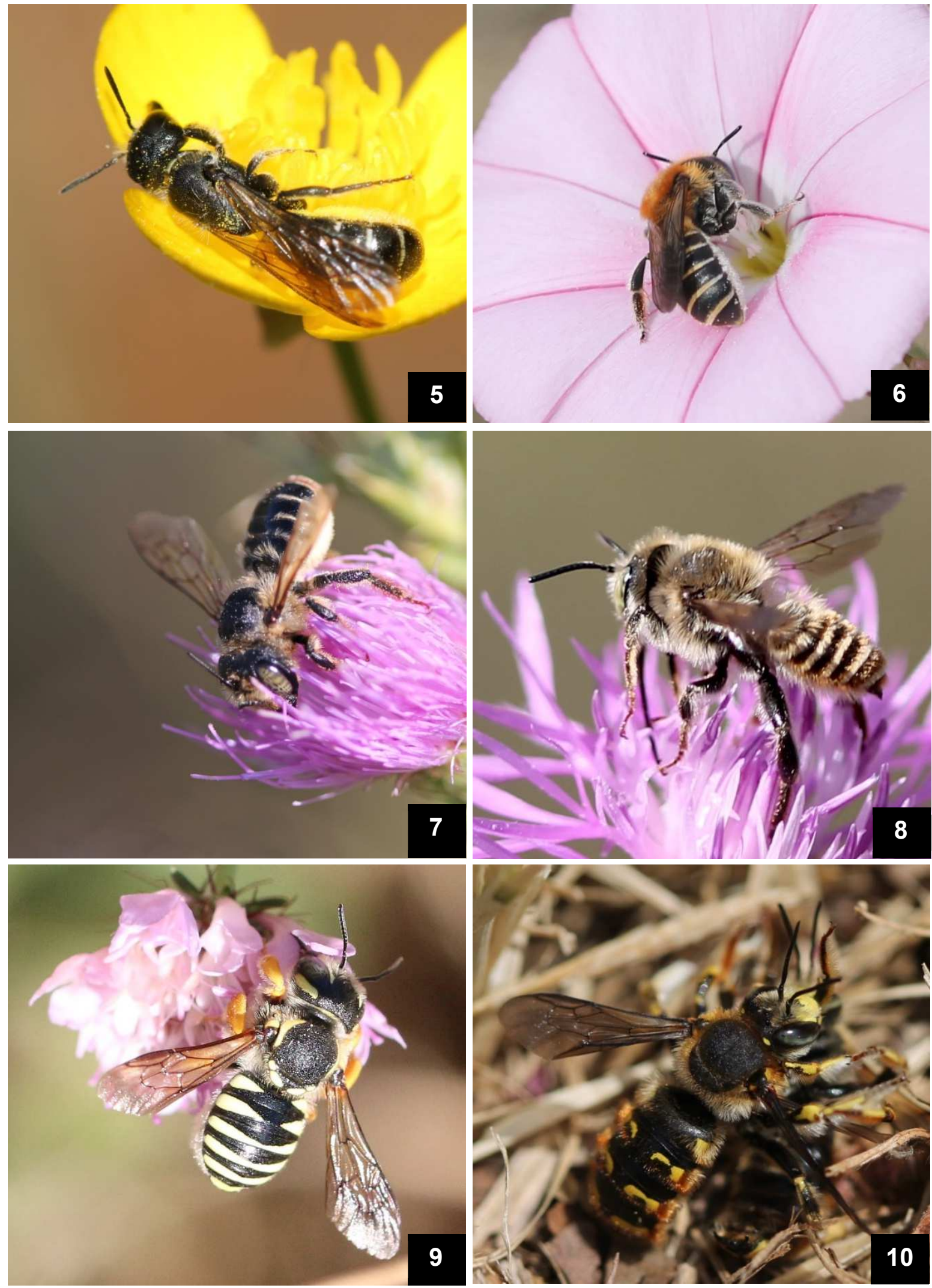

Figures 5-10. 5. Chelostoma florisomne (MORAWITZ, 1868), sur Bouton d'or (Saint-Paul-de-Loubressac, 06/06/2017). 6. Hoplitis perezi (FERTON, 1895), sur Liseron (Creysse, 07/06/2015). 7. Lithurgus chrysurus (FonsCOLOMBE, 1834), sur Centaurée sp. (Le Montat, 11/07/2018). 8. Lithurgus chrysurus (FonsCOLOMBE, 1834), sur Chardon (Cahors, 04/09/2018). 9. Trachusa interrupta (FABRICIUS, 1781), sur Scabieuse colombaire (Cieurac, 16/07/2017). 10. Anthidium manicatum (LiNNAEUS, 1758) défendant son territoire contre un autre mâle de la même espèce (Floirac, 07/06/2015) 

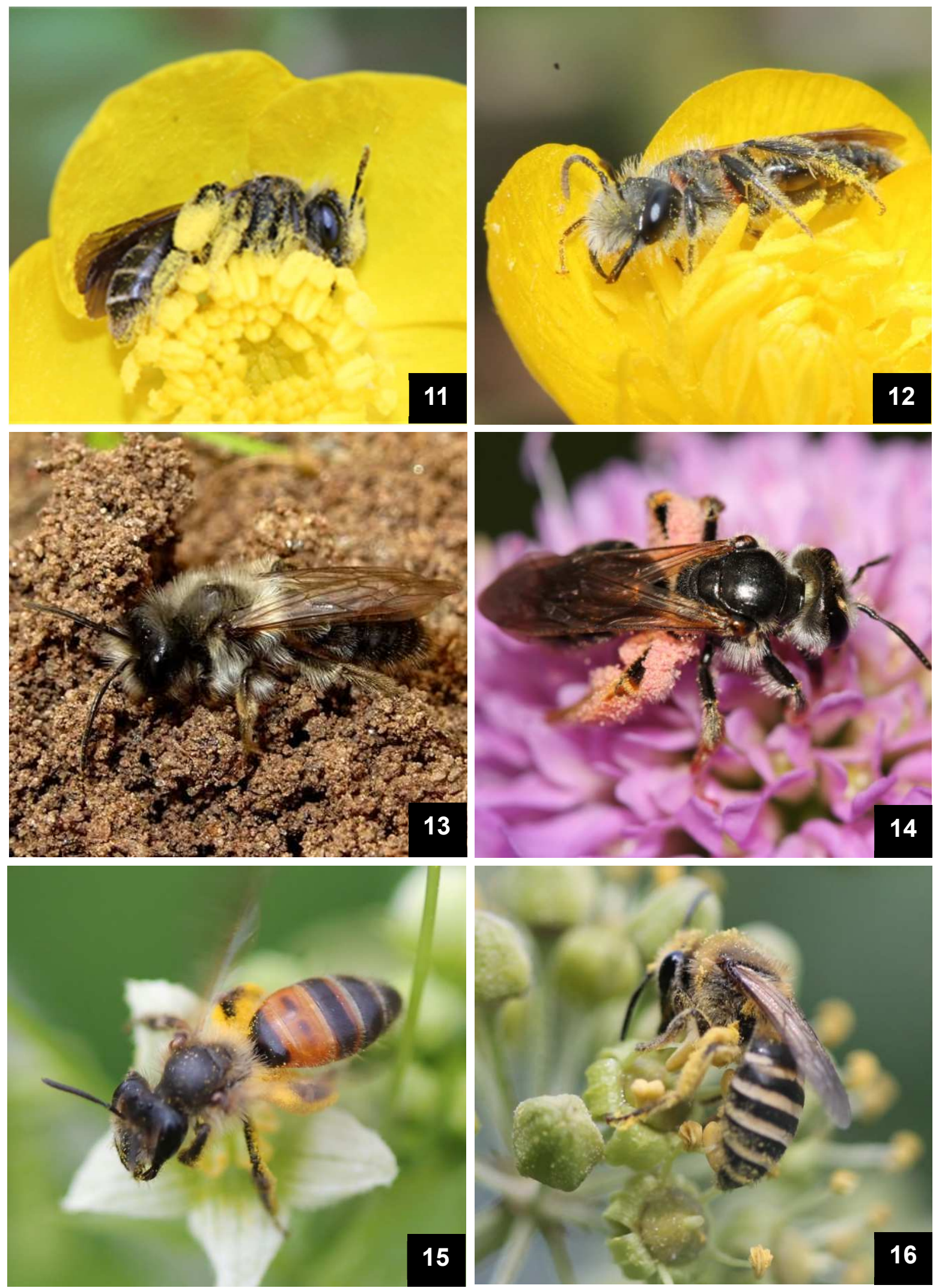

Figures 11-16. 11-12. Andrena ranunculi (SCHMIEDEKNECHT, 1883) (11: Femelle; 12 : Mâle), sur Bouton d'or (Le Montat, 27/04/2017). 13. Andrena vaga (PANZER, 1799), sur site de nidification (Pradines, 02/04/2018). 14. Andrena hattorfiana (FABRICIUS, 1775), sur Scabieuse (Sousceyrac, 29/05/17). 15. Andrena florea (FABRICIUS, 1793), sur Bryone (Cahors, 01/06/2015). 16. Colletes hederae (SCHMIDT \& WESTRICH, 1993), sur fleur de Lierre (Lavercantière, 11/10/2015). 

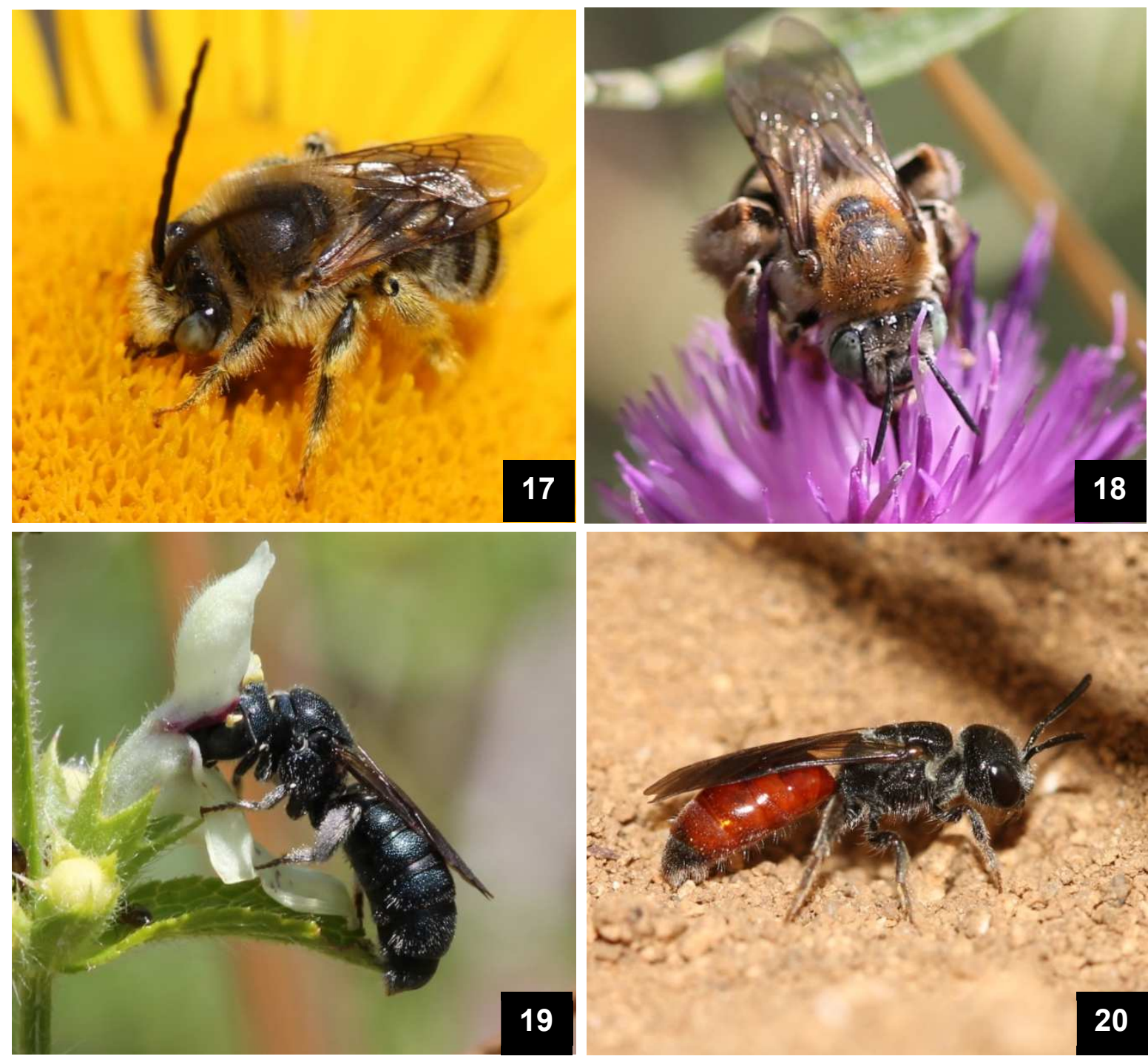

Figures 17-20. 17. Tetraloniella fulvescens (GIRAUD, 1863), sur Inule (Rocamadour, 27/06/2016). 18. Tetraloniella dentata (Germar, 1839), sur Centaurée (Payrignac, 11/08/2015). 19. Ceratina gravidula (GERSTÄCKER, 1869), sur stachys (Cieurac, 27/06/2016). 20. Sphecodes monilicornis (KIRBY, 1802), au sol (Salviac, 29/05/2017).

\section{Remerciements}

Un grand merci à mon regretté ami Gérard CANOU (décédé en décembre 2017) qui m'a permis d'accéder à sa collection d'Anthophila du Lot d'avant 1965, ainsi qu'à Marc et Hubert TUSSAC qui m'ont donné accès et transmis leurs collections d'Anthophila lotois. Je tiens à remercier également le CEN Midi-Pyrénées (Samuel DANFLOUS) pour le matériel de spécimens du Lot transmis jusqu'à présent. Ma gratitude va également à Benoît GESLIN pour son aide précieuse et sa collaboration en tant que directeur de la publication de la revue Osmia. Je remercie également tous les spécialistes experts de l'Observatoire des Abeilles (OA) pour les déterminations d'espèces effectuées tout au long des dernière années ; dans l'ordre alphabétique : Matthieu AubERT, Éric DufRÊNE, David GEnOUd, Gérard LE GofF, Gilles MAHÉ ainsi que Serge GADOUM (OPIE). Je remercie aussi les spécialistes étrangers pour leurs déterminations d'espèces : Holger DATHE pour les Hylaeus, Mickael KuHLMANn pour les Colletes, Alain PAULY pour les Halictidae et Stephan RISCH pour les Eucerinii.

Enfin, je tiens à remercier bien sincèrement mon ami, Henri Savina (spécialiste des Symphytes, Région Occitanie), pour son aide précieuse et ses remarques pertinentes sur les premières versions de cet article. 
Bibliographie

AMiet F, 1996. Insecta helvetica 12. Fauna Hymenoptera, Apidae, 1. Teil. Schweizerischen Entomologischen Gesellschaft, Zürich (Confédération helvétique), et Centre Suisse de Cartographie de la Faune, Neûchatel (Confédération helvétique), 98 pp.

Amiet F, Müller A \& Neumeyer R, 1999. Fauna helvetica 4. Apidae 2. Schweizerischen Entomologischen Gesellschaft, Zürich (Confédération helvétique), et Centre Suisse de Cartographie de la Faune, Neûchatel (Confédération helvétique), 219 pp.

Amiet F, Herrmann M, Müller A \& NEUMEYER R (2001). Fauna helvetica 6. Apidae 3. Schweizerischen Entomologischen Gesellschaft, Zürich (Confédération helvétique), et Centre Suisse de Cartographie de la Faune, Neûchatel (Confédération helvétique), 208 pp.

Amiet F, Herrmann, Müller A \& Neumeyer R 2004. Fauna helvetica 9. Apidae 4. Schweizerischen Entomologischen Gesellschaft, Zürich (Confédération helvétique), et Centre Suisse de Cartographie de la Faune, Neûchatel (Confédération helvétique), 273 pp.

Amiet F, Herrmann M, Müller A \& NEUMEYER R 2007. Fauna helvetica 20. Apidae 5. Schweizerischen Entomologischen Gesellschaft, Zürich (Confédération helvétique), et Centre Suisse de Cartographie de la Faune, Neûchatel (Confédération helvétique), 356 pp.

Amiet $\mathbf{F}$, Herrmann $M$, Müller A \& NeUMEYer R 2010. Fauna helvetica 26. Apidae 6. Schweizerischen Entomologischen Gesellschaft, Zürich (Confédération helvétique), et Centre Suisse de Cartographie de la Faune, Neûchatel (Confédération helvétique), $317 \mathrm{pp}$.

Amiet F, MÜller A \& NeUmeyer R, 2014. Fauna helvetica 4. Apidae 2. $2^{\text {e }}$ édition revue et corrigée (1 $\mathrm{e}^{\mathrm{e}}$ édition 1999). Schweizerischen Entomologischen Gesellschaft, Zürich (Confédération helvétique), et Centre Suisse de Cartographie de la Faune, Neûchatel (Confédération helvétique), 239 pp.

Amiet F, Müller \& Praz C. (2017). Fauna helvetica 29. Apidae 1. $2^{2}$ édition revue et corrigée (1e édition 1996). Schweizerischen

Entomologischen Gesellschaft, Zürich (Confédération helvétique), et Centre Suisse de Cartographie de la Faune, Neûchatel (Confédération helvétique), $187 \mathrm{pp}$.

AnONYME, 2020. Territoires: Gestion de l'espace: Cartographie. Chambre d'agriculture du Lot, Cahors (France).

https://lot.chambre-

agriculture.fr/territoires/gestion-de-

lespace/cartographie/ (consulté en 2019)

APOIDEA ARMORICANA, 2018. Listes départementales des abeilles de Bretagne, Pays-dela-Loire et Basse Normandie. Observatoire des Abeilles.

https://oabeilles.net/les-abeilles-de-france

(consulté en 2019)

Baliteau L, Iserbyt S, Mahé G, RASMont P, Le Goff G, Pauly A \& Scheuchl E, 2013. Contribution à l'inventaire des Abeilles sauvages du département de l'Aveyron (France) (Hymenoptera, Apoidea). Bulletin de la Société entomologique de France, 118 (3) : 343-362.

https://www.persee.fr/doc/bsef_0037-

928x 2013 num 11833116

BANASZAK J \& ROMASENKO L, 2001. Megachilid bees of Europe (Hymenoptera, Apoidea, Megachilidae). Pedagogical University of Bydgoszcz (Pologne), 239 pp.

Benoist R, 1931. Les Osmies de la faune française. Annales de la Société entomologique de France, 100 : 23-60.

BÉnon D \& Praz C, 2016. Deux nouvelles espèces d'abeilles sauvages pour l'entomofaune suisse : observation d'Andrena florivaga EVERSMANN, 1852 et Andrena fulvicornis SCHENCK, 1853 dans les pâturages jurassiens, Entomo Helvetica, 9 : 69-78.

https://sciencesnaturelles.ch/uuid/d3590825-56e95881-b1e5-

a6b5b952be17? $\mathrm{r}=20170706115333 \quad 1502366030 \quad 3$ d1c6c76-9cf2-5c23-abee-e47475b665c3

(consulté en juillet 2020)

Bischoff I, Feltgen K \& BRECKNer D, 2003. Foraging strategy and pollen preferences of Andrena vaga (PANZER) and Colletes cunicularius 
(L.) (Hymenoptera: Apidae). Journal of Hymenoptera Research, 12 (2) : 220-237.

https://doi.org/10.5962/bhl.part.241

BLÜTHGEN P, 1924. Contribución al conocimiento de las especies españolas de 'Halictus' (Hymenoptera Apidae). Memorias de la Real Sociedad Española de Historia Natural, 11 (9) : 332-544.

BLÜTHGEN P, 1951. Neues oder Wissenswertes über mitteleuropäische. Aculeaten und Goldwespen II (Hym.). Bonn zoological Bulletin früher Bonner Zoologische Beiträge, 2 (3-4) : 229234.

BWARS, 2019. BWARS Bees, Wasps \& Ants Recording Society. Site internet de l'association. https://www.bwars.com/home

(consulté en 2019)

Chambers V H, 1949. The Hymenoptera Aculeata of Bedfordshire. Transactions of the Society for British Entomology, 9: 197-252.

Danforth B N, FAng J \& Sipes S D, 2006. Analysis of family-level relationship in bees (Hymenoptera: Apiformes) using 28S and two previously unexplored nuclear genes: CAD and RNA polymerase II. Molecular Phylogenetics and Evolution, 39: 358-372.

https://doi.org/10.1016/j.ympev.2005.09.022

DANFORTH BN, SiPES SD, FANG J \& BRAdy SG, 2006b. The history of early bee diversification based on five genes plus morphology. Proceedings of the National Academy of Sciences of the United States of America, 103: 15118-15123.

https://doi.org/10.1073/pnas.0604033103

Dorchin A, López-Uribe MM, Praz CJ, GRISWOLD T \& DANFORTH BN, 2018. Phylogeny, new generic-level classification, and historical biogeography of the Eucera complex (Hymenoptera: Apidae). Molecular Phylogenetics and Evolution, 119: 81-92.

https://doi.org/10.1016/j.ympev.2017.10.007

DUFRÊne É, GENOUd D \& BourLeT P, 2016. Sur la distribution en France de Lithurgus cornutus FABRICIUS 1827 (Hymenoptera - Megachilidae Lithurgini) : apport de données récentes. Osmia, $6: 16-21$. https://oabeilles.net/wp-

content/uploads/2016/10/OSMIA_6P_Article_Lcor nutus_Eric_Dufr\%C3\%AAne.pdf

(consulté en 2019)

Dusmet y Alonso JM, 1908. Los Ápidos de España. III. Gen. Anthidium, Memorias de la Real Sociedad Española de Historia Natural, 5 (4) : 153-214.

Ebmer AW, 1975. Neue Westpaläarktische Halictidae (Halictinae, Apoidea). Teil III. Linzer biologische Beiträge, 7 (1) : 41-118.

https://www.zobodat.at/pdf/LBB_0007_1_00410118.pdf

Ebmer AW, 1988. Kritische Liste der nichtparasitischen Halictidae Österreichs mit Berucksichtigung aller mitteleuropaïschen Arten (Insecta: Hymenoptera: Apoidea: Halictidae). Linzer biologische Beiträge, 20 (2): 527-711.

https://www.zobodat.at/pdf/LBB_0020_2_05270711.pdf

FrIESE H, 1897. Monographie der Bienengattung Nomia (Latr.) (Palaearctische Formen). FestSchrift zur Feier des fünfzigjahrigen Bestehens des Vereins für schlesische Insektenkunde in Breslau, 1897 : 45-84.

FRIESE H 1922. Die europäischen Bienen (Apidae) - Das Leben und Wirken unserer Blumenwespen. Eine Darstellung der Lebensweise unserer wilden wie gesellig lebenden Bienen nach eigenen Untersuchungen für Naturfreunde, Lehrer $u$. Zoologen. Vereinigung Wissenschaftlicher Verleger, Berlin \& Leipzig, 122 pp +7 pls.

Gargominy O, Tercerie $\mathrm{S}$ ， RÉGNier $\mathrm{C}$, RAMAgE T, DUPONT $\mathbf{P}$, DASZKIEWICZ $\mathbf{P} \&$ PONCET L, 2018. TAXREF v12, référentiel taxonomique pour la France : Méthodologie, mise en ouvre et diffusion. Muséum national d'histoire naturelle, Paris, 62 pp.

https://www.google.com/url?sa=t\&rct=j\&q=\&esrc $=$ s\& source $=$ web\&cd $=\& c a d=$ rja\&uact $=8 \& v e d=2 a h$ UKEwi015Twp7vqAhVV9IUKHSXyAREQFjABe gQIAhAB\&url=https $\% 3 \mathrm{~A} \% 2 \mathrm{~F} \% 2 \mathrm{Finpn} . \mathrm{mnhn}$.fr\% 2Fdocsweb $\% 2 \mathrm{~F}$ docs $\% 2 \mathrm{~F}$ download $\% 2 \mathrm{~F} 239729 \&$ usg=AOv Vaw24F9a9A0NBfteYjmnLK7O2

(consulté en 2019) 
GEBHARDT M \& RöHR G, 1987. Zur Bionomie der Sand-bienen Andrena clarkella (KIRBY), $A$. cineraria (L.), A. fuscipes (KIRBY) und ihrer Kuckucksbienen (Hymenoptera: Apoidea). Drosera, 87, 896-114.

GENOUD D, 2012. Analyse des peuplements d'Hyménoptères Apoidea de la réserve naturelle de la Massane à partir du matériel entomologique disponible. Réserve naturelle de la Masanne. Travaux, $95:$ : 1-36.

http://www.rnnmassane.fr/wpcontent/uploads/travaux/T95.pdf (consulté en 2019)

GENoud D, 2017. Synthèse des connaissances sur les Hyménoptères Apoidea. Prélèvements 20022009 et étude 2014. Réserve naturelle de la Masanne. Travaux, 108 : 1-56.

http://www.rnnmassane.fr/wpcontent/uploads/2017/09/T108.pdf

(consulté en 2019)

GoGala A, 1999. Bee fauna of Slovenia: Checklist of species (Hymenoptera: Apoidea). Scopolia, 42: 1-79.

http://www.dlib.si/stream/URN:NBN:SI:DOC-

OE2SHYYW/2a 7ba685-376e-423c-a453-

e5ebde7969b1/PDF

(consulté en 2019)

GuSEnLEITNER F, SchWARZ M, 2002. Weltweite Checkliste der Bienengattung Andrena mit Bemerkungen und Ergänzungen zu paläarktischen Arten (Hymenoptera, Apidae, Andreninae, Andrena). Entomofauna, Supplement $12: 1-1280$.

https://www.zobodat.at/pdf/ENTS_S12_00011280.pdf

Klein A M, Vaissière B, CANe J H, SteffanDewenter I, Cunningham S A, Kremen C \& TSCHARNTKE T 2007. Importance of pollinators in changing landscapes for world crops. Proceedings of the Royal Society B, 274 : 303-313.

https://doi.org/10.1098/rspb.2006.3721

KOCOUŘEK K, 1966. Prodromus der Hymenopteren der Tschechoslowakei. Part 9: Apoidea, 1. Acta Faunistica Entomologica Musei Nationalis Prague, 12 (Supplement): 1-122.
KuHLMANN M (ed.) (2015). Checklist of the Western Palaearctic Bees (Hymenoptera: Apoidea: Anthophila). Site personnel.

http://westpalbees.myspecies.info

[dernière consultation le 25 juin 2020]

LACHAUD A \& MAHÉ G, 2008. Contribution à la connaissance de la diversité des abeilles sauvages de Loire-Atlantique. Bretagne vivante - SEPNB, $91 \mathrm{pp}$.

LIEFTINCK MA, 1983. Notes on the nomenclature and synonymy of Old World Melectine and Anthophorine bees (Hymenoptera, Anthophoridae). Tijdschrift voor Entomologie, 126 (12): 269-284.

https://www.biodiversitylibrary.org/part/66050\#/s ummary

Michener CD, 2007. The bees of the world. 2nd ed. John HoPKINs Univ. Press, Baltimore, Maryland, USA, 913 pp.

Michez, D. (2007). La nouvelle classification des abeilles (Hymenoptera, Apoidea, Apiformes) ou la chute de l'abeille mellifère (Apis mellifera L.) de son piédestal. Osmia, 1: 23-26.

Michez D, Terzo M \& Rasmont P, 2004. Phylogénie, biogéographie et choix floraux des abeilles oligolectiques du genre Dasypoda LATREILLE 1802 (Hymenoptera : Apoidea : Melittidae). Annales de la Société entomologique de France (n. s.), 40 (3-4) : 421-435.

https://doi.org/10.1080/00379271.2004.10697431

Michez D \& Patiny S, 2005. World revision of the oil-collecting bee genus Macropis PANZER 1809 (Hymenoptera, Apoidea, Melittidae) with a description of a new species from Laos. Annales de la Société entomologique de France (n. s.), 41 (1) : 15-28.

https://doi.org/10.1080/00379271.2005.10697439

Michez D \& EARDLEY CD, 2007. Monographic revision of the bee genus Melitta KIRBY 1802 (Hymenoptera: Apoidea: Melittidae). Annales de la Société entomologique de France (n. s.), 43 (4) : 379-440.

https://doi.org/10.1080/00379271.2007.10697535 
MÜLLER A, 1996. Host-Plant Specialization in Western Palearctic Anthidine Bees (Hymenoptera: Apoidea: Megachilidae). Ecological Monographs, 66 (2) : 235-257.

https://doi.org/10.2307/2963476

MÜLler A, 2019. Palaearctic Osmiine Bees, ETH Zürich. http://blogs.ethz.ch/osmiini (consulté en 2019)

MÜLLER A \& Kuhlmann M, 2008. Pollen hosts of western palaearctic bees of the genus Colletes (Hymenoptera: Colletidae): the Asteraceae paradox. Biological Journal of the Linnean Society, 95 : 719-733.

https://oi.org/10.1111/j.1095-8312.2008.01113.x

NiETO A, ROBERTS SPM, KEMP J, RASMONT P, Kuhlmann M, García Criado M, BiesmeiJer JC, Bogusch P, DAThe HH, De la RÚa P, DE Meulemeester $T$, Dehon $M$, Dewulf $A$, Ortiz-SÁnchez $\mathbf{F}$ J, Lhomme P, Pauly A, Potts S G, Praz C, Quaranta M, Radchenko V G, Scheuchl E, Smit J, Straka J, Terzo M, TomoziI B, Window J \& Michez D, 2014. European Red List of Bees. Publication Office of the European Union, Luxembourg, 84 pp.

https://dx.doi.org/10.2779/77003

NoskiewICZ J, 1936. Die paläarktischen ColletesArten. Prace Naukowe Wydawnictwo Towarzystwa Naukowego we Lwowie, 3 : 1-531.

OBSERVATOIRE DES ABEILlES, 2020. Observatoire des Abeilles. Site de l'association, Flines-lez-Raches (France).

https://oabeilles.net

(consulté en 2019)

PAXTON RJ \& PoHL H, 1999. The tawny mining bee, Andrena fulva (MÜLLER) Hymenoptera, Andreninae), at a south Wales field site and its associated organisms: Hymenoptera, Diptera, Nematoda and Strepsiptera. British Journal of Entomology and Natural History, 12 : 57-67.

https://www.biodiversitylibrary.org/part/94566\#/s ummary

Peeters TMJ, Raemakers I P \& Smit J, 1999. Voorlopige atlas van de Nederlandse bijen (Apidae). European Invertebrate Survey Nederland, Leiden, 230 pp.
Pérez J, 1879. Contribution à la faune des Apiaires de France. Actes de la Société linnéenne de Bordeaux, 33 : 119-229.

PÉreZ J, 1886 (1883). Contribution à la faune des Apiaires de France. Deuxième partie. Actes de la Société linnéenne de Bordeaux, 37 : 205-378, pls 34.

Pérez J, 1890. Catalogue des mellifères du SudOuest. Actes de la Société linnéenne de Bordeaux, $44: 133-200$.

Perkins R C L, 1919. The British species of Andrena and Nomada. Transactions of the Entomological Society of London, 1919: 218-320, pls XI-XV.

Petit J, 1986. Sur les Mégachilides du GrandDuché de Luxembourg (Hymenoptera, Megachilidae). Lambillionea, 86 (3-4) : 31-39.

Philippe C, 2020. Lise préliminaire des Abeilles sauvages du département du Lot. Observatoire des Abeilles, Flines-lez-Raches (France), 9 pp.

https://oabeilles.net/wp-

content/uploads/2020/03/Tableau-occurrence-desabeilles-sauvages-du-LOT 290320.pdf

$\underline{\mathrm{OU}}$ http://www.osmia-journal-

hymenoptera.com/uploads/1/3/2/6/132680733/tabl eau-occurrence-des-abeilles-sauvages-du-

lot_290320.pdf (consultation juillet 2020)

PraZ C J, 2017. Subgeneric classification and biology of the leafcutter and dauber bees (genus Megachile Latreille) of the western Palearctic (Hymenoptera, Apoidea, Megachilidae). Journal of Hymenoptera Research, 55 : 1-54.

https://doi.org/10.3897/jhr.55.11255

RASmont P, Ebmer A, BANASzaK J, VAN DER ZANDEN G 1995. Hymenoptera Apoidea Gallica. Liste taxonomique des abeilles de France, de Belgique, de Suisse et du Grand-Duché de Luxembourg. Bulletin de la Société entomologique de France, 100 (HS) : 1-98.

https://www.researchgate.net/publication/259781 448_Hymenoptera_Apoidea_Gallica_Liste_taxono mique des abeilles de France de Belgique de Suisse_et_du_Grand-Duche_de_Luxembourg 
Rasmont P, Aubert M, Dufrêne É, Genoud D, Lair X, Le Goff G, Mahé G, Michez D, Terzo M \& VereeCKeN $\mathbf{N}$, 2016. Fichier de référence pour la faune d'Apoidea d'Europe. Fichier Excel REFERENTIEL transmis par P. Rasmont le 16/06/2016.

Rasmont P, Genoud D, Gadoum S, Aubert M, Dufrêne É, LE Goff G, MAhÉ G. Michez D \& Pauly P, 2017. Hymenoptera Apoidea Gallica. Liste des abeilles sauvages de Belgique, France, Luxembourg et Suisse. Atlas Hymenoptera, Université de Mons, Mons, Belgium, 15 pp.

http://applications.umons.ac.be/docnum/c7b423fdd183-486c-9cec-966066b9b364/611CA728-5E704 A5C-8743-

247E54A8D46F/414_Rasmont_et_al_2017_Hyme noptera_Apoidea_Gallica_2017_02_16.pdf

(consulté en 2019)

RASMONT P, FRANZÉN M, LECOCQ T, HARPKE A, Roberts S P M, BiesmeiJer K, Castro L, Cederberg B, Dvořk L, Fitzpatrick U, Gonseth Y, HaUbruge É, MAHÉ G, Manino A, Michez D, Neumayer J, ØDegaARd $F$, Paukkunen J, PaWlikowski T, Potts S G, Reemer M, SetTele J, Straka J, SchWeiger O, 2015. Climatic Risk and Distribution Atlas of European Bumblebees. Biorisk, 10 (special issue): $246 \mathrm{pp}$.

https://doi.org/10.3897/biorisk.10.4749

Rust R W, Cambon G, Torre Grossa J P, VAISSIÈRE B E, 2004. Nesting Biology and Foraging Ecology of the Wood-boring Bee Lithurgus chrysurus (Hymenoptera: Megachilidae). Journal of the Kansas Entomological Society, 77 (3): 269-279.

https://www.jstor.org/stable/25086215

SCHMid-EGGer C, \& SCHEUCHL E, 1997. Illustrierte Bestimungstabellen der Wildbienen Deutschlands und Österreichs unter Berücksichtigung der Arten der Schweiz. Band III. Andrenidae. Apollo Books, Stenstrup (Danemark), $180 \mathrm{pp}$.

SCHEUCHL E \& Willner W 2016. Taschenlexikon der Wildbienen Mitteleuropas Alle Arten im Porträt. Quelle \& Meyer, Wiebelsheim (Allemagne), $917 \mathrm{pp}$.

SCHINDLER M \& WitTMANN D, 2004. Interaktionen zwischen Kuckucksbienen der
Gattung Nomada und ihren Wirten (Andrena) : Labor und Freilanduntersuchungen. Beiträge der Hymenopteren-Tagung in Stuttgart : 18.

SCHMID-EGGER C, 2011. Hymenoptera Aculeata from "Parc national du Mercantour" (France) and "Parco delle Alpi Marittime" (Italy) in the southwestern Alps. Ampulex, 3 : 13-50.

http://www.ampulex.de/ampu3.pdf

(consulté en 2019)

SchmidT K \& Westrich P, 1993. Colletes hederae n. sp., eine bisher unerkannte, auf Efeu (Hedera) spezialisierte Bienenart (Hymenoptera: Apoidea). Entomologische Zeitschrift, 103: 89-93.

SChMiedeKnecht O, 1882. Apidae Europaeae (Die Bienen Europas) per genera, species et varietates dispositae atque descriptae. Nomada, Bombus, Psithyrus et Andrena. 1. Publié par l'auteur, Berlin, 866 pp.

SchWARZ M, 1966. Zur Kenntnis der Gattung Nomada ScOP. (Hymenoptera, Apoidea). Nachrichtenblatt der Bayerischen Entomologen, 15 (3-4): 17-24.

SChWARZ M, GUSENLEITNER F, Westrich P \& DATHE H H, 1996. Katalog der Bienen Österreichs, Deutschlands und der Schweiz (Hymenoptera, Apidae). Entomofauna, Supplement $8: 1-398$.

https://www.zobodat.at/pdf/ENTS_S8_00010398.pdf

Sedivy C, Dorn S, Widmer A \& Müller A 2013. Host range evolution in a selected group of osmiine bees (Hymenoptera: Megachilidae): the Boraginaceae-Fabaceae paradox. Biological Journal of the Linnean Society, 108 : 35-54.

https://doi.org/10.1111/j.1095-8312.2012.02013.x

SITDIKov AA, 1988. Sistematika pcheli roda Eucera Scopoli (Hymenoptera, Anthophoridae) fauny SSSR i sopredel'nykh stran: podrod Pteneucera Tkalcu [Systematics of the genus Eucera SCOPOLI (Hymenoptera, Anthophoridae) of the USSR and neighbour countries Subgenus Pteneucera TKALCUீ]", Trudy zoologicheskogo Instituta AN SSSR, 175: 102-111 [en russe].

Smit J, 2004. De wespbijen (Nomada) van Nederland (Hymenoptera: Apidae). Nederlandse Faunistische Mededelingen, 20: 33-125. 
https://www.repository.naturalis.nl/record/261964

SMIT J. 2018. Identification key to the European species of the bee genus Nomada SCOPOLI, 1770. Entomofauna, Monographie 3 : 253 pp. https://www.zobodat.at/pdf/ENT M3 00010253.pdf

StandFuss K \& SchwarZ M, 2007. Zur aktuellen Bienenfauna der Ölbaumzone in SOThessalien / Griechenland (Hymenoptera : Apoidea: Apiformes). 2. Die parasitischen Bienen (pro parte : Apidae, Megachilidae, Halictidae). Entomofauna, 28 (24) : 293-320.

https://www.zobodat.at/pdf/ENT_0028_02930320.pdf

STÖckHeRT F K, 1933. Die Bienen Frankens (Hym. Apid.). Eine ökologisch-tiergeographische Untersuchung. Beiheft Deutsche Entomologische Zeitschrift, 1932 : 1-294.

Straka J \& Bogusch P 2007. Phylogeny of the bees of the family Apidae based on larval characters with focus on the origin of cleptoparasitism (Hymenoptera: Apiformes). Systematic Entomology, 32 : 700-711. https://doi.org/10.1111/j.1365-3113.2007.00394.x

UICN France (2018). Guide pratique pour la réalisation de Listes rouges régionales des espèces menacées - Méthodologie de l'UICN \& démarche d'élaboration. Seconde édition. Paris, $60 \mathrm{pp}$.

https://uicn.fr/wp-content/uploads/2018/04/guidepratique-listes-rouges-regionales-especes-

menacees.pdf

(consulté en 2019)

Vereecken N, Toffin E, Gosselin M \& MicheZ D, 2006. Observations relatives à la biologie et à la nidification de quelques abeilles psammophiles d'intérêt en Wallonie. 1. Observations printanières. Parcs \& Réserves, 61 (1) : 8-13.

WARNCKE K, 1967. Faunistische Bemerkungen über westpaläarktische Bienen der Gattung Andrena F. (Hymenoptera, Apoidea). Bulletin de l'Institut Agronomique et des Stations de Recherches de Gembloux, 2 (3) : 569-581.
WARNCKE K, 1983. Zur Kenntnis der Bienengattung Pasites JURINE, 1807, in der Westpaläarktis (Hymenoptera, Apidae, Nomadinae). Entomofauna, 4 (21) : 261-347. https://www.zobodat.at/pdf/ENT_0004_0261$\underline{\text { 0347.pdf }}$

WARNCKE K, 1992. Die westpaläarktischen Arten der Bienengattung Sphecodes LATR. (Hymenoptera, Apidae, Halictinae). Bericht der Naturforschenden Gesellschaft Augsburg, 52 : 964.

https://www.zobodat.at/pdf/Ber-Naturf-GesAugsburg 0521992 0009-0064.pdf

WeStrich P, 1989. Die Wildbienen BadenWürttembergs. Ulmer, Stuttgart, 972 p. [2nd ed. published 1990].

Müller A, (2019). Palaearctic Osmiine Bees. ETH Zürich.

https://blogs.ethz.ch/osmiini/

(consulté en 2019)

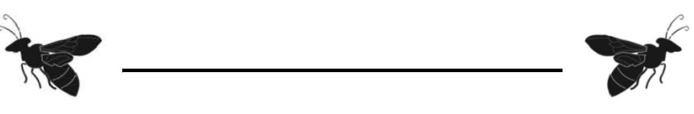

